\title{
Attribute Reduction Algorithm Based on Discernibility Matrix with Algebraic Method
}

\author{
GAO Jing ${ }^{1, a}$, Ma Hui ${ }^{1}$, Han Zhidong ${ }^{2, b}$ \\ ${ }^{1}$ Information School, Capital University of Economics and Business, Beijing 100070 \\ ${ }^{2}$ Beijing Information Center of China UnionPay Co., Ltd, Beijing 100193 \\ aemail:gaojinggaoj@126.com, bemail:hanzhidong@sina.com
}

Keywords: Rough Set; Positive Region; Discernibility Matrix; Algebra Method; Complexity

\begin{abstract}
There are many algorithms of attribute reduction based on discernibility matrix, where the element of the discernibility matrix is used as heuristic information. But there are few algebra methods about attribute reduction based on discernibility matrix. In this paper, we proposed an algorithm of attribute reduction with algebra method. First of all, we should design an algorithm to compute the heuristic information. Then we used this heuristic information to design an new algorithm of attribute reduction based on discernibility matrix, whose time complexity is $\mathrm{O}\left(|\mathrm{C}|^{2}|\mathrm{U}|\right)$, and whose space complexity is $\mathrm{O}(|\mathrm{U}|)$. At last, we used an example to illustrate the new algorithm's validity and high efficiency.
\end{abstract}

\section{Introduction}

Attribute Reduction is one of the researches in rough set theory ${ }^{[1]}$, and which based on discernibility matrix has attracted a lots of attention as its simplify and intuitive ${ }^{[2]}$. On one hand, the research on Attribute Reduction Algorithm Based on Discernibility Matrix is more inclined to be designed by the heuristic information, which is composed by elements of the discernibility matrix. On the other hand, Professor Pawlak has put forward many design methods of Attribute Reduction Algorithm of Attribute Reduction model. Namely, they are respectively algebra method ${ }^{[3]}$, discernibility matrix method ${ }^{[4-6]}$ and matrix ${ }^{[7]}$.etc.

So is attribute reduction algorithm of attribute reduction model based on information entropy proposed by Professor Wang, however, But the design of attribute reduction algorithm of discernibility matrix in algebraic method is less used, this is mainly because the model has no direct algebraic definition

Reference [10-11] illustrates a new attribute reduction model based on information. Reference [12] has proved that the model is equal to the Attribute Reduction Algorithm Based on Discernibility Matrix. Of which Reference [13] has put forward a matrix method. So, the paper designs an algebra method of Attribute Reduction based on discernibility. The Specific steps are as follows: Firstly, we design a algorithm to calculate a heuristic information with rapid response, then based on the heuristic information we design Attribute Reduction algorithm based on discernibility matrix. Whose time complexity is $\mathrm{O}\left(|\mathrm{C}|^{2}|\mathrm{U}|\right)$, and space complexity is $\mathrm{O}(|\mathrm{U}|)$. At last, an example is provided to illustrate the new algorithm.

\section{Basic Theory}

Definition 1: Decision Table is defined as follows: $S=(U, C, D, V, f, d)$. In this formula, $U=\left\{x_{1}, x_{2}, \cdots, x_{n}\right\}$ is the domain, $C=\left\{c_{1}, c_{2}, \cdots, c_{r}\right\}$ is the conditions attribute sets. $D$ is the decision attribute sets. $f: U \times C \rightarrow V$ and $d: U \times D \rightarrow V$ is information functions, in the functions, $F=C \cup D, C \cap D=\varnothing, V=\cup V_{a}, a \in F, V_{a}$ means range of a.

Definition 2:

Define discernibility matrix as $M=\left(m_{i j}\right)$ in $S=(U, C, D, V, f, d)$. The element is as follows: 


$$
m_{i j}=\left\{\begin{array}{l}
\left\{c_{k} \mid c_{k} \in C, f\left(x_{i}, c_{k}\right) \neq f\left(x_{j}, c_{k}\right), d\left(x_{i}, D\right) \neq d\left(x_{j}, D\right)\right\} \\
\varnothing \text { else }
\end{array}\right.
$$

In this formula, $k=1,2, \ldots, r ; i, j=1,2, \cdots, n$.

Definition 3: In decision table, $S=(U, C, D, V, f, d)$, we hypothesize that $M=\left(m_{i j}\right)$ is $\mathrm{HU}$ discernibility matrix, $\forall B \subseteq C$, if $B$ satisfies following conditions: (1) $\forall \varnothing \neq m_{i j} \in M$, $B \cap m_{i j} \neq \varnothing ;(2) \forall b \in B, B-\{b\}$ doesn't satisfy (1), $B$ is considered as attribute reduction based on discernibility matrix . all attribute reductions is short for $\operatorname{HRED}_{C}(D)$.

Definition 4: in decision table, $S=(U, C, D, V, f, d)$, any attribute in $\mathrm{U} \quad B \subseteq C \cup D$ ( knowledge, Equivalence relation family, $U / B=\left\{B_{1}, B_{2}, \cdots, B_{t}\right\}$ ) is a random variable consisted in subset of $U$ in algebra., the Probability distribution is confirmed by the following methods:

$$
[B: p]=\left[\begin{array}{cccc}
B_{1} & B_{2} & \cdots & B_{t} \\
p\left(B_{1}\right) & p\left(B_{2}\right) & \cdots & p\left(B_{t}\right)
\end{array}\right]
$$

In the formula, $p\left(B_{j}\right)=\left|B_{j}\right| /|U|, j=1,2, \cdots, t$

\section{Definition 5:}

In decision table, $S=(U, C, D, V, f, d)$, the knowledge information of decision attributes sets $U / D=\left\{D_{1}, D_{2}, \cdots, D_{k}\right\}$ respecting to condition attributes sets $U / C=\left\{C_{1}, C_{2}, \cdots, C_{m}\right\}$ is defined as follows:

$$
\begin{aligned}
& I(D \mid C)=\sum_{i=1}^{m} \sum_{j=1}^{k}\left|\frac{X_{i} \cap D_{j}}{|U|}\right|\left|\frac{D_{j}}{|U|}-\frac{X_{i} \cap D_{j}}{|U|}\right| \\
& X_{i}^{j}=X_{i} \cap D_{j} \in X_{i} / D=\left\{X_{i}^{1}, X_{i}^{2}, \cdots X_{i}^{k}\right\}
\end{aligned}
$$

Definition 6:

In decision table, $S=(U, C, D, V, f, d)$, for $\forall b \in B \subseteq C$, if $I(D \mid B)=I(D \mid(B-\{b\}))$, b is Omitted for B with respect to $\mathrm{D}$. or, $\mathrm{b}$ is cannot be omitted for $\mathrm{B}$ with respect to $\mathrm{D}$. for $\forall B \subseteq C$, if any element is necessary for $\mathrm{D}$, then $\mathrm{B}$ is independent for $\mathrm{D}$.

Definition 7:

In decision table, $S=(U, C, D, V, f, d)$, if $\forall B \subseteq C, I(D \mid B)=I(D \mid C)$ and $\mathrm{B}$ is independent for $D$, then $B$ is attribute reduction based on knowledge for $C$ with respect to $D$. mark all the attribute reduction as $N e w R E D_{C}(D)$.

Theorem 1, in the decision table, $S=(U, C, D, V, f, d)$, Attribute reduction based on discernibility matrix is equivalent to attribute reduction based on amount of knowledge.

We will design attribute reduction based on discernibility matrix with algebraic method under the condition of Theorem 1 ;

Theorem 2, in the decision table, $S=(U, C, D, V, f, d), \forall C \supseteq A \supseteq B, I(D \mid A) \leq I(D \mid B)$ is deduced.

Definition 8: in the decision table, $\forall a \notin C-B$, define the heuristic information as $\operatorname{sig}_{B}(a)=I(D \mid B)-I(D \mid B-\{a\})$.

Obviously, $\operatorname{sig}_{B}(a) \geq 0$, and $\operatorname{sig}_{B}(a)$ get bigger, the incremental amount of information of the a property is greater

Definition 9:

In the decision table, $S=(U, C, D, V, f, d) \quad, \quad \forall X \subseteq U, X /\{a\}=\left\{X_{1}, X_{2}, \cdots X_{r}\right\}$, $g(X, a)=\sum_{i=1}^{r} \sum_{j=1}^{k}\left|\frac{X_{i} \cap D_{j}}{|U|}\right| \frac{X_{i} \cap D_{j}}{|U|}-\frac{X \cap D_{j}}{|U|} \mid$ is the function we define.

Theorem 3, in the decision table, $S=(U, C, D, V, f, d), \forall U / B=\left\{B_{1}, B_{2}, \cdots, B_{t}\right\}, a \notin C-B$, 
$\operatorname{sig}_{B}(a)=\sum_{i=1}^{t} g\left(B_{i}, a\right)$ can be deduced from definition 8 and definition 9.

\section{High-efficient attribute reduction algorithm}

In order to get the high-efficient attribute reduction algorithm, firstly we get the algorithm of quick response calculation heuristic information.

Algorithm1: calculate $g(X, a)$

Input: decision table $S=(U, C, D, V, f, d), \forall X \subseteq U, a \in C$

Output: $g(X, a)$

1). Calculating $X /\{a\}$ with Radix sort in the reference [3]

2).calculating $X / D, X_{i} / D$ with radix sort in the reference [3]

3).calculating $g(X, a)$ with the formula in definition 9.

Complexity analysis of algorithm 1 :

The time complexity of the first step of algorithm is $O(|X|)$, The time complexity of second step is $O(|X|)$, so the time complexity of algorithm is $O(|X|)$, The worst-case complexity of space is $O(|X|)$.

Algorithm 2: calculate $\operatorname{sig}_{B}(a)$.

Input: in decision table, $S=(U, C, D, V, f, d), \forall U / B=\left\{B_{1}, B_{2}, \cdots, B_{t}\right\}, a \in C-B$

Output: $\operatorname{sig}_{B}(a)$.

1) calculate $g\left(B_{i}, a\right)$ with algorithm 1 .

2) calculate $\operatorname{sig}_{B}(a)$ with algorithm 3 .

Complexity principal analysis of algorithm 2:

The time Complexity of the first step of algorithm is $O\left(\left|B_{i}\right|\right)$, so the worst-case complexity of time is $\sum_{i=1}^{t} O\left(\left|B_{i}\right|\right)=O(|U|)$, the worst-case complexity of space is $O(|U|)$.

Algorithm 3: attribute reduction algorithm

Input: decision table $S=(U, C, D, V, f, d)$.

Output: attribute reduction $\mathrm{R}$;

1) $R=\varnothing$;

2) for any $a \in C-R$, calculate $\operatorname{sig}_{R}(a)$ with algorithm 2 .

3) make $\operatorname{sig}_{R}(b)=\max _{a \in C-R}\left|\operatorname{sig}_{R}(a)\right|$, if $\operatorname{sig}_{R}(b)>0$, then $R=R \cup\{b\}$, get to the step 2, or get to the step 4 .

4) output the attribute reduction $R$.

Complexity principal analysis of algorithm 3:

The worst-case time complexity of second step of algorithm 3 is $O(|C-R \| U|)$, so the worst-case time complexity of algorithm 3 is $\sum_{|R|=0}^{|C|} \sum_{i=1}^{t} O(|C-R \| U|)=O\left(|C|^{2}|U|\right)$, the worst-case space complexity is $O(|U|)$.

\section{Example analysis}

Decision table in the literature [13] is used to illustrate the new algorithm. 
Table 1 decision table

\begin{tabular}{|l|l|l|l|l|l|}
\hline & $\mathrm{a}$ & $\mathrm{b}$ & $\mathrm{c}$ & $\mathrm{d}$ & $\mathrm{D}$ \\
\hline $\mathrm{X} 1$ & 2 & 1 & 2 & 1 & 0 \\
\hline $\mathrm{X} 2$ & 2 & 2 & 2 & 1 & 1 \\
\hline $\mathrm{X} 3$ & 2 & 1 & 2 & 1 & 0 \\
\hline $\mathrm{X} 4$ & 2 & 2 & 2 & 1 & 1 \\
\hline $\mathrm{X} 5$ & 3 & 1 & 2 & 1 & 0 \\
\hline $\mathrm{X} 6$ & 1 & 2 & 3 & 2 & 2 \\
\hline
\end{tabular}

Calculate $\operatorname{sig}_{\varnothing}(a)=\frac{2}{6}\left(\frac{0}{6}+\frac{0}{6}\right)+\frac{2}{6}\left(\frac{0}{6}+\frac{0}{6}\right)+\frac{1}{6}\left(\frac{2}{6}+\frac{2}{6}+\frac{1}{6}\right)+\frac{1}{6}\left(\frac{2}{6}+\frac{2}{6}+\frac{1}{6}\right)=\frac{10}{36}$ with algorithm 3 . By the same token, $\operatorname{sig}_{\varnothing}(b)=\frac{18}{36}, \operatorname{sig}_{\varnothing}(c)=\frac{10}{36}=\operatorname{sig}_{\varnothing}(d)$. We choose attribute b according the third step, so we get the equation $R=\{b\}$, and then get to the step 2. Calculate $\operatorname{sig}_{\{b\}}(a)=\frac{8}{36}, \operatorname{sig}_{\{b\}}(c)=\operatorname{sig}_{\{b\}}(d)=\frac{4}{36}$, we choose attribute 2 according to the third step. So we get the expression- $R=\{b, a\}$, then jump to step 2, calculate $\operatorname{sig}_{\{b, a\}}(c)=\operatorname{sig}_{\{b, a\}}(d)=0$ by step 4 , attribute reduction $R=\{b, a\}$ is outputted.

\section{Conclusion}

In this paper, algebraic expression attribute reduction definitions based on discernibility matrix is put forward, and then starting from the attribute reduction definitions of algebraic methods, the heuristic information is defined, and the algorithm of heuristic information calculating with rapid reaction is provided, finally design an attribute reduction algorithm based on discernibility matrix, witch time complexity is $O\left(|C|^{2}|U|\right)$ and space complexity is $O(|U|)$. Finally, an example is used to illustrate the effectiveness and efficiency of the new algorithm.

\section{Acknowledgement}

In this paper, the research was supported by the Beijing talented persons training scientific research project in 2012 (Project name: Algorithm of data mining of incomplete information systems ; Project No.2012D005019000001 ); the research was supported by the importation and development of high-caliber talents project of Beijing Municipal Institutions in 2013(Project No.CIT\&TCD201304135;Project name: Decision tree generation algorithm and its optimization of incomplete information systems); the research was supported by Beijing Education Committee funds on how to improve science research level; the research was supported by National Natural Science Foundation of China (Grant No. 61303018).

\section{References}

[1] Pawlak Z. Rough Sets [J]. International Journal of Computer and information Science, 1982, 11(5): 341-356

[2] Hu Xiao Hua, Cercone N. Learning in relational databases: a rough set approach [J]. Computational Intelligence, 1995, 11(2):323-337

[3] Zhang Yan $\mathrm{Xu}$, Zuo Peng Liu, Bing Ru Yang, Wei Song. A Quick Attribute Reduction Algorithm with Complexity of $\max (\mathrm{O}(|\mathrm{C}||\mathrm{U}|), \mathrm{O}(|\mathrm{C}| 2|\mathrm{U} / \mathrm{C}|))[\mathrm{J}]$. Chinese Journal of Computers, 2006, 29 (3) : 391-399

[4] Jia Yang Wang, Can Gao. Improved Algorithm for Attribute Reduction Based on Discernihility Matrix [J]. Computer Engineering, 2009, 35(3): 66-69 
[5] Dong Yi Ye, Zhao Jun Chen. A New Discernibility Matrix and the Computation of a Core [J]. Acta Electronica Sinica, 2002,30(7):1086-1088

[6] Ming Yang, Zhi Hui Sun. Improvement of Discernibility Matrix and the Computation of a Core [J]. Journal of Fudan University (Natural Science), 2004, 43(5):865-868

[7] Zhang Yan Xu. Research on Attribute Reduction Algorithm Based on Rough Sets [D]. University of Science \& Technology Beijing, 2008,1

[8] Guo Yin Wang, Yu Hong, Da Chun Yang. Decision Table Reduction based on Conditional Information Entropy [J].Chinese Journal of Computers, 2002, 25( 7) :759-76

[9] GuoYin Wang. Rough reduction in algebra view and information view[J]. International Journal of Intelligent System, 2003(18): 679-688

[10] Jiye Liang, K.S.Chin, Chuangyin Dang, Richard C.M.Yam. A new method for measuring uncertainty and fuzziness in rough set theory[J]. International Journal of General Systems,2002,31(4):331-4342

[11] Ping Luo, Qing He, Zhongzhi Shi. Theoretical study on a new information entropy and its use in attribute reduction[C], ICCI,2005,73-79

[12]Zhangyan Xu, Bingru Yang, Wei Song,Wei Hou. Illustrating the Attribute Reduction Based on HU's Discernibility Matrix with Information View [J]. Computer Science, 2007, 34(9):191-193

[13] Ren Meng, Zhangyan Xu, Bingru Zhang. Matrix Description for Attribute Reduction Based on Skowron Discernibility Matrix [J]. Computer Engineering, 2010, 36(17):54-57. 